\title{
Short tutorial. Treatment options in light chain amyloidosis and monoclonal gammopathy of renal significance
}

\author{
Maria T. Krauth · Hermine Agis $(\mathbb{D}$
}

Received: 20 January 2021 / Accepted: 20 January 2021 / Published online: 3 February 2021

(C) The Author(s) 2021

\begin{abstract}
Summary AL amyloidosis (AL) and monoclonal gammopathy of renal significance (MGRS) are both paraprotein-associated diseases. Both entities are based on a monoclonal paraprotein produced by a clonal plasma-cell population like in monoclonal gammopathy of undetermined significance (MGUS) or by a Bcell population like in low grade lymphoma. Per definition MGUS and low-grade lymphoma do not require treatment. But in rare cases the monoclonal M-gradient acts as a "toxic" protein inducing severe multimodal organ damage as in AL and MGRS. Urgent treatment is indicated in AL and in MGRS to avoid irreparable loss of organ function or death. No treatment is currently approved in Europe for AL or MGRS. On January 15, 2021 the US Food and Drug Administration approved the monoclonal anti-CD38 antibody for treatment of AL. To minimize the serum M-gradient concentration, a clone directed therapy as in multiple myeloma or B-cell malignancies treatment regimens can be applied. In AL, an additional treatment option is under investigation. These special drugs are directed against the typical amyloid-fibrils responsible for deposition formation. An additional and important consideration in this special field of rare diseases is the option of organ transplantation in particular kidney transplantation in MGRS. All these treatment modalities are addressed in this article.
\end{abstract}

Keywords Clone-directed treatment .

Antifibrillary antibody · Proteasome inhibitor . Immunomodulatory drugs (IMiDs) · Monoclonal antibody · Autologous stem cell transplantion (ASCT)

\footnotetext{
M. T. Krauth $\cdot$ H. Agis $(\bowtie)$

Division of Hematology and Hemostaseology, Department Internal Medicine I, Medical University Vienna, Währinger Gürtel 18-20, 1090 Vienna, Austria

hermine.agis@meduniwien.ac.at
}

\section{Introduction}

In AL and MGRS, tissue and organ damage are linked to a monoclonal paraprotein [1]. In AL the pathomechanism of tissue and organ damage is well-characterized. A monoclonal misfolded protein with reduced solubility is produced by either a clonal B-cell population or by a clonal plasma-cell population. This paraprotein, most often clonal free-light-chains, form insoluble fibrils and lead to extracellular depositions. These "toxic" depositions induce tissue damage with loss of organ function (Figs. 1 and 2).

In MGRS [2], renal tissue damage is also linked to a monoclonal paraprotein but the pathomechanisms are very diverse and multimodal [3]. Different forms of deposition diseases such as monoclonal immunoglobulin deposition disease (MIDD), light-chain deposition disease (LCDD), and heavy-chain deposition diseases (HCDD) are defined but additional modes of damage are clarified in light-chain proximal tubulopathy (LCPT), light-chain cast nephropathy, cryoglobulinemia or C3-nephropathy.

Because of the high diversity of pathomechanisms and the multitude of paraprotein-associated lesions, a classification system for MGRS was introduced in 2019 [3].

\section{Therapeutic options}

Up to January 15, 2021 no approved treatment was available in this special field of rare diseases. Robust data from prospective randomized phase III studies were lacking because of the low incidence of these rare diseases. For improvement and restoration of organ function and prolongation of overall survival specialized treatment is of major importance. In AL two main strategies are under investigation [4]. One group of treatment options is based on clonal directed reg- 

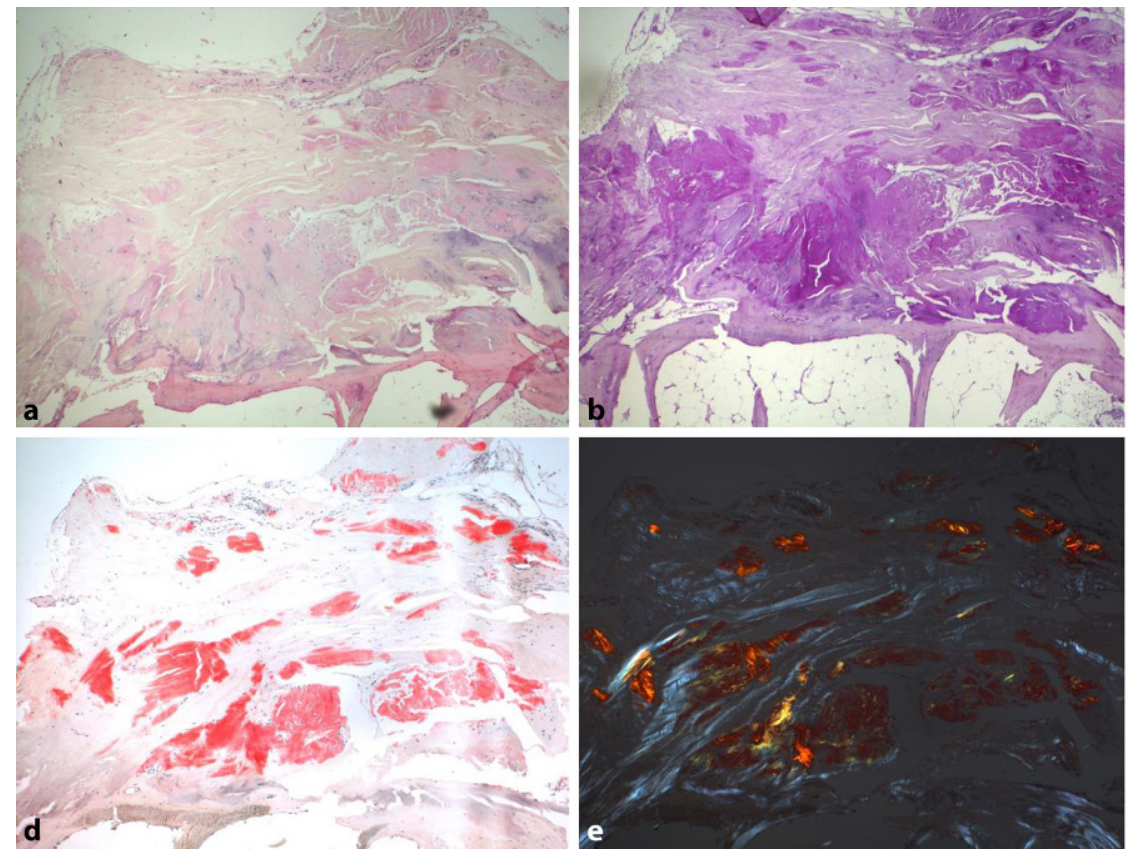

Fig. 1 Different staining techniques from a bone marrow biopsy specimen with kappa light-chain amyloidosis. a $\mathrm{H}+\mathrm{E}$ staining, b PAS staining, c Giemsa staining, d Congo red staining, e Congo red with polarized light. (Images kindly pro-

imens well-known from multiple myeloma care. Reducing the number of clonal B-cells or clonal plasma cell is associated with reduction of the "toxic" M-protein. This strategy is effective in AL and in MGRS [5]. The other strategy is a very specialized form of treatment based on antiamyloid-fibril directed drugs [6] and is applicable only in AL.

\section{Clone directed drugs}

Clone directed drugs are well known from antimyeloma therapy. In multiple myeloma these regimens are well defined and controlled in randomized phase III trials. Based on the low incidence of AL (and MGRS) only a very small number of prospective controlled randomized of phase III trials are available. In these rare trials it could be documented that treatment and response to treatment improves overall survival [7]. When antimyeloma drugs are administered in $\mathrm{AL}$ these drugs are accompanied by a very different and more severe toxicity-profile. This should be considered especially if advanced cardiac involvement is present [7].

\section{Proteasome inhibitors}

Targeting the proteasome is highly effective in treating plasma cell diseases. The amyloidogenic light chain is a highly effective stressor that sensitizes plasma cells to proteasome inhibitor (PI) toxicity. Especially in AL the plasma-cell population is highly sensitive against PI-associated stress. This special mode of PI-associ- vided by Univ. Prof. Dr. Ingrid Simonitsch-Klupp, Department of Pathology, Medical University Vienna). PAS periodic-acid Schiff reaction, $H \& E$ hematoxyline \& eosin

ated action is postulated as drug-induced aggravation of a pre-existing intracellular proteotoxic stress induced by the amyloidogenic light chain itself. The misfolded light chains propagate severe intracellular dysregulation in protein trafficking and protein degradation leading to severe vulnerability of the clonal plasma cells and enhanced apoptosis [8].

\section{Bortezomib}

Bortezomib is currently the most widely used PI within the treatment of plasma cell-associated AL. Bortezomib is given as single agent or in doublet or triplet combinations. Dexamethasone and alkylating agents (melphalan, cyclophosphamide) are frequently combined with bortezomib.

In AL the subcutaneous application of bortezomib seems to be preferable, in a once-weekly schedule, and in a risk-adapted dosage [9, 10]. Bortezomib should be used with caution in particular if cardiac involvement is present [11]. Bortezomib is suspected to induce cardiotoxicity. In renal impairment no dose-adjustment is necessary. If peripheral polyneuropathy is pre-existing the use of bortezomib is not recommended [12].

The most common regimen is cyclophosphamide, bortezomib and dexamethasone (CyBorD) in various risk-adapted schedules and dosages [13]. In particular in patients with AL and renal impairment CyBorD is the first choice since no dose adjustment is required. The overall hematologic response rates in the two large trials with CyBorD were 60-65\% [14]. 


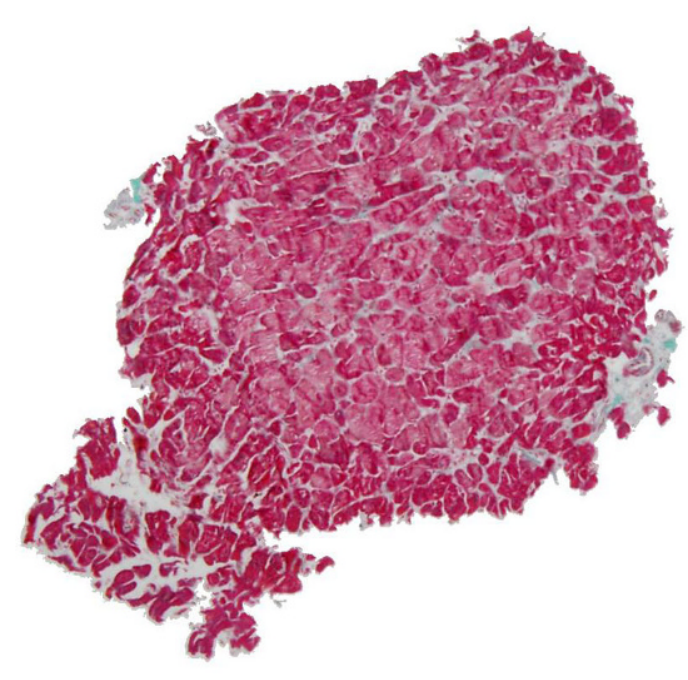

a

Fig. 2 Endomyocardial biopsy specimen from a donor with only minimal extracellular matrix deposition and a patient with high volume depositions during lambda light chain amyloidosis and cardiac involvement. Stained with modified trichrome staining technique: red cardiomyocytes, green extracellular

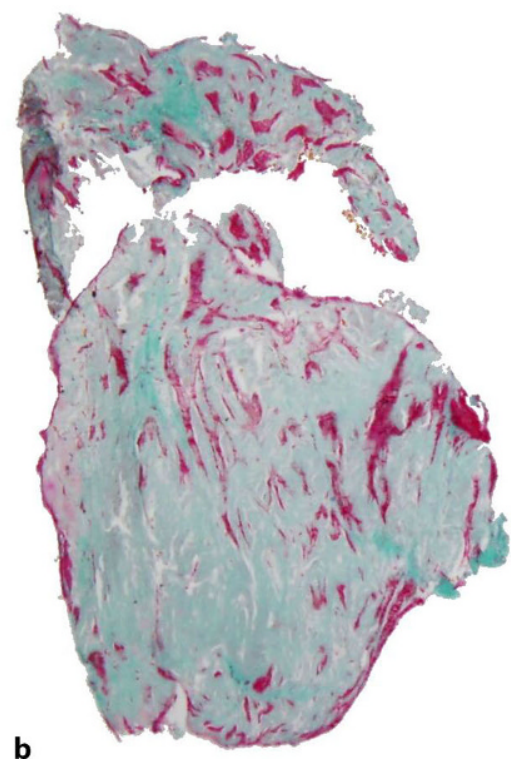

matrix. a Donor specimen with mostly cardiomyocytes and only minimal extracellular matrix deposition; b patient with high volume extracellular matrix deposition and only very few cardiomyocytes. (Images kindly provided by Dr. Franz Duca, Department of Cardiology, Medical University Vienna)

In patients with $\mathrm{AL}$, carfilzomib should be handled with caution. Significant cardiac, pulmonary and renal toxicities are documented, in particular in AL with multiorgan involvement. In a small group of patients with peripheral neuropathy without cardiac depositions carfilzomib was well tolerated and highly active [20].

\section{Ixazomib}

Ixazomib is the only PI with oral intake and very low neurotoxicity. In a small phase I/II study in relapsed/refractory AL amyloidosis (RRAL), single agent activity of ixazomib was demonstrated [21]. Based on the promising data from this small study an international randomized controlled phase III trial (TOURMALINE-AL1) ixazomib/dexamethasone compared to physician's choice treatment (except bortezomib-based regimens) in patients with 1-2 prior lines of therapy [22]. In this trial ixazomib/ dexamethasone was not associated with higher rates of hematologic responses (53 vs. $51 \%$ ) and failed to meet the primary end point. Some important secondary end points were reached including time to vital organ deterioration or death (35 vs. 26 months) and hematologic CR rate (26 vs. $18 \%$ ). The hematologic responses were long lasting (46.5 vs. 20.2 months) in the ixazomib/dexamethasone arm versus the physician's choice arm. Vital organ PFS was also longer in the ixazomib/dexamethasone arm (18 vs. 11 months) [22].

If ixazomib is the more favorable PI in newly diagnosed AL with higher efficacy or a better safety profile has to be addressed in further trials. 


\section{Immunomodulatory drugs}

The immunomodulatory drugs (IMiD) category is represented by three drugs: thalidomide, lenalidomide and pomalidomide. All three derivatives are taken orally. In multiple myeloma the IMiDs are widely used in first line regimens as well as in the relapsed/ refractory multiple myeloma (RRMM) setting. IMiDbased therapy is accompanied by significant toxicities: fatigue, skin rashes, thromboembolic events, infections, renal and/or cardiac deterioration. In systemic AL with cardiac and renal involvement thalidomide and lenalidomide-containing regimen are also associated with reduced tolerability. A common phenomenon within all IMiDs is the paradoxical increase in NTproBNP with and without clinical deterioration (reversible after drug cessation). Timely response assessment is clearly complicated during IMiD-based regimens.

\section{Thalidomide}

Thalidomide is accompanied by significant toxicities especially in AL with cardiac involvement (bradycardia and heart rhythm disturbance). In AL with preexisting PNP thalidomide should be avoided.

\section{Lenalidomide}

Lenalidomide is mainly tested in RRAL cohorts. The ORR did not exceed 50\% in most studies. Deep hematologic response rates were also rare and subsequently the organ responses were also poor. The lenalidomide-associated toxicities were significant in AL with renal involvement and also in patients with cardiac impairment. Vigorous dose-adjustments were essential to improve tolerability and reduce treatment-induced organ deterioration [23]. The median lenalidomide dosage was $10 \mathrm{mg}$ per day (maximum dose $15 \mathrm{mg}$ per day). Treatment discontinuation were observed frequently.

\section{Pomalidomide}

Pomalidomide is approved in RRMM as third-generation IMiD. Pomalidomide is known for the ability to overcome lenalidomide and bortezomib-resistance in RRMM. No dose-adjustment is required in patients with renal insufficiency. Recently rapid hematologic responses are described in RRAL if treated with pomalidomide and dexamethasone (at least partial response rate 44\%) [24]. In most of the patients a first response was observed after one cycle of therapy. Hematologic responses translated into improved overall survival (median survival 50 vs. 27 months). At least partial hematologic responses resulted in significant longer progression-free survival (PFS) rates (median PFS 37 vs. 18 months). Grade 3 adverse events were documented in $33 \%$ of patients. Mainly infectious complications (9\%), cardiac failure $(7 \%)$, increase in creatinine $(6 \%)$ and cytopenia $(2 \%)$ were reported [24].

\section{Monoclonal antibody-based treatment options}

\section{Antiplasma cell-directed antibodies}

Daratumumab Daratumumab is a monoclonal, fully humanized IgG1 kappa antibody directed against CD38 with promising efficacy in relapsed/refractory AL. Even in heavily pretreated AL overall hematologic response rates between 55-90\% were reported [25]. The hematologic responses were accompanied by improvement of organ function and better overall survival [26]. In all daratumumab cohorts low toxicity and good tolerability were reported [26]. High tolerability and efficacy are of utmost importance in particular in systemic AL with pronounced cardiac involvement and multiorgan impairment.

Currently an international phase III trial (ANDROMEDA Trial, NCT03201965) was initiated. Dara s.c. + CyborD (daratumumab subcutaneous plus cyclophosphamide, bortezomib, dexamethasone) is compared with CyBorD in the frontline setting. The data from the safety run-in phase were presented with excellent $96 \%$ overall response rate within the Dara-CyBorD arm and $82 \%$ achieved a very good partial response (VGPR) [27]. The ANDROMEDA trial data were presented to the US Food and Drug Administration (FDA) and on January 15, 2021 daratumumab was approved for treatment of AL by the FDA. An additional daratumumab first-line trial is ongoing focusing on ultrahigh-risk AL stage IIIB.

Isatuximab Isatuximab is also a monoclonal antiCD38 antibody approved for treatment in relapsed refractory multiple myeloma. The efficacy of isatuximab in relapsed refractory AL [28] and in MGRS [29] is currently under investigation.

Elotuzumab Elotuzumab is a monoclonal IgG antibody directed against SLAM-F7 (signaling lymphocytic activation molecule F7). From multiple myeloma trials it is known that elotuzumab does not exhibit single agent activity. It needs an IMiD partner for antimyeloma/antiplasma cell activity. The effectivity of elotuzumab combined with lenalidomide and dexamethasone in AL is under investigation.

\section{Antiamyloid-directed drugs}

\section{Antifibrillar antibodies}

Antifibrillar antibodies are directed against amyloid fibrils. These artificial monoclonal antibodies are created for rapid clearance of amyloid depositions in vital organs and reversing of end-organ damage.

\section{NEOD001 (birtamimab)}

NEOD001 is a humanized monoclonal anti-IgG1 antibody. This antibody is directed against repeating epitopes of AL amyloid fibrils. The binding of the NEOD001 to the amyloid-forming fibrils induces de- 
position destabilization, macrophage attraction and activation. These mechanisms are shown to enhance amyloid degradation and clearance of amyloid deposits [30].

Remarkable data were published for the antifibrillar antibody NEOD001 in 2016 and 2017. A phase I/II study documented good tolerability and effectiveness concerning the hematological response as well as the improvement of the organ functions (heart, kidneys and nerves) [31]. A clear improvement in organ function could be seen in $53 \%$ of the patients $(n=36)$ with cardiac involvement and $63 \%$ of the patients $(n=35)$ with renal involvement. A phase III study was then initiated (VITAL study) and patients with AL (cardiac, renal, hepatic, neurogenic) were randomized double-blind into two arms. All patients received a bortezomib-based therapy either in combination with NEOD001 or with a placebo. No statistically significant differences between NEOD001 + "standard of care" vs. placebo + "standard of care" could be detected at time of primary end point evaluation (12 months after trial start) [32]. The PRONTO study comparing NEOD001 vs. placebo in patients with systemic AL with persistent cardiac dysfunction was also a negative study. Because of these negative results further drug development was discontinued and the studies were terminated in April 2018 due to futility [32].

In the follow-up observations the patients with severe cardiac amyloidosis Mayo stage IV demonstrated a clear benefit from treatment with NEOD001 compared to placebo. In the light of these recent results further investigations are warranted in this special population with the highest risk of early death and dismal outcome.

\section{1-1F4 (CAEL 101)}

CAEL 101 acts as antiamyloid-fibril directed monoclonal antibody (IgG1-kappa). In a first in human phase $1 \mathrm{a} / \mathrm{b}$ trial excellent tolerability with no doselimiting toxicity was demonstrated [33].

A total of 27 patients with relapsed AL were included; 24 were evaluated. In all, 18 patients had cardiac and/or renal involvement. Twelve patients (67\%) showed an improvement in organ function; none of the patients showed signs of progression during treatment with CAEL 101. The median time to the first measurable response was 3 weeks. It is noteworthy that the improvement in organ function developed independently of the level of free light chains in the serum [33]. Based on these highly promising results two phase III trials with CAEL 101 will start in the near future: A Study to Evaluate the Effectiveness and Safety of CAEL-101 in Patients with Mayo Stage IIIa AL Amyloidosis (ClinicalTrials.gov identifier: NCT04512235) and A Study to Evaluate the Effectiveness and Safety of CAEL-101 in Patients With Mayo Stage IIIb AL Amyloidosis (ClinicalTrials.gov identifier: NCT04504825).

\section{Antiserum amyloid $P$ directed drug combination} (dezamizumab, miridesap)

Serum amyloid $\mathrm{P}$ (SAP) is a ubiquitous plasma protein without fully clarified function. On the other hand SAP is an important nonfibrillar component of all amyloid deposits independent of the primary origin of the amyloid forming protein. These two highly different modes of action are addressed by a highly specialized anti-SAP directed drug-combination. This combination is based on a unique small molecule (CPHPC, miridesap) required for serum depletion of the SAP molecules and the second drug is of a fully humanized IgG 1 monoclonal antibody (dezamizumab). The monoclonal anti-SAP antibody is directed against the SAP-protein fixing amyloid-fibrils within the depositions [34]. A small phase I trial was published in 2015 [35]. The treatment with the anti-SAP drugcombination resulted in a significant reduction of the amyloid load within 6 weeks in AL as well as in transthyretin amyloidosis (ATTR). However, patients with cardiac and renal amyloidosis were excluded from the start. Only patients with amyloidosis of the liver, spleen or lymph nodes were recruited. In an expanded cohort the drug combination was tested in patients with cardiac involvement [36]. Two adverse events were observed-hypotension and erythema multiforme-like rush. No substantial other adverse events were documented. In the consecutive phase II trial, including patients with cardiac and renal involvement, launched in February 2017, a change in the risk/benefit profile was observed and the trial and drug development were stopped and terminated [37].

\section{Small molecules}

\section{Venetoclax}

Venetoclax is an oral BCL2 inhibitor approved for CLL-treatment. Venetoclax has also been investigated in relapsed multiple myeloma and has shown promising results in patients with $t(11 ; 14)$ [38]. Surprisingly a higher mortality rate was found in the venetoclax cohort in the phase III BELLINI trial in multiple myeloma patients, contradicting the superior responses and progression-free survival results. The reasons of the high death rate were severe infectious complications within the venetoclax arm. The BELLINI trial was set on hold because of these findings.

In AL $50-60 \%$ of patients harbor translocation $\mathrm{t}(11 ; 14)$ [39]. Preliminary results from case reports suggest high effectiveness of venetoclax in relapsed AL. In a systemic retrospective multicenter analysis of 24 patients with relapsed AL and venetoclax-containing regimens the overall response rate (ORR) was $75 \%$ [40]. For the patients with $t(11 ; 14)$ the ORR was $86.7 \%$ and in patients negative for $\mathrm{t}(11: 114)$ the ORR was $55.6 \%$. A phase I trial was initiated for further 
investigations of venetoclax in relapsed AL patients. This trial has been suspended because of the severe infectious adverse events within the BELLINI trial in multiple myeloma [38].

\section{Doxycycline}

Doxycycline is known for his antifibrillary activity [41] in patients with AL and is an effective treatment option. A retrospective study on 76 patients treated upfront with a bortezomib-based regimen revealed a significant better 1-year survival rate in patients treated additionally with doxycycline compared to those without doxycycline. In the doxycycline group, there was an improvement in cardiac function in $60 \%$ of the patients, compared with $18 \%$ in the control group with a 1-year survival advantage of 82 vs. 53\% [42]. Doxycycline is currently under further investigation.

\section{Green tea compound epigallactocatechin-3-gallate}

The green tea compound epigallactocatechin-3-gallate (ECGC) is suspected to have the ability to disrupt amyloid fibril formations. These data are collected in preclinical trials. Within the clinical setting one randomized trial (TAME-AL, operated in Germany) did not show any benefit of EGCG on reducing left ventricular wall thickness over placebo [43].

\section{Autologous blood stem cell transplant (ASCT)}

ASCT is one of the most effective options in AL treatment. The main problem up to now is, that only $25-30 \%$ of patients diagnosed with AL are eligible for this procedure. ASCT in AL is described by Priv. Doz. Dr. Alexandra Böhm in this issue.

\section{Organ transplantation}

\section{Heart transplantation in $A L$}

The overall survival of AL patients is mainly determined by the severity of cardiac involvement. With extensive cardiac involvement, early death between 3-12 months after diagnosis is frequent. The serious problems within these group of frail patients are based on the life-threatening cardiac amyloid deposits and on severe side effects of the treatment itself. If cardiac involvement is so severe that starting of any therapy is impossible, heart transplantation can be an option [44].

Heart transplantation (HTX) should be offered to strictly selected patients and in specialized centers. A main criterion is that only a single organ should be affected. Age and comorbidities are two main decisive arguments within decision-making. If possible, induction treatment should be administered before the HTX procedure in order to reduce or eliminate the "toxic" paraprotein. The main purpose of the HTX is to improve the severe impaired cardiac function to allow further AL therapy and ASCT. This implies ASCT after HTX [44]. At present it is undefined when consecutive ASCT should be performed after HTX. This concept is highly demanding and should be applied in specialized centers.

\section{Kidney transplantation}

Organ transplantation in particular kidney transplantation (NTX) is a burning question in AL and in MGRS. In AL kidney involvement is documented in around $70 \%$. In $15-30 \%$ of the patients terminal kidney failure occurs during the course of disease independent of the response to treatment [45].

New therapeutic options have significantly improved response rates and overall survival times. Recently, a single center specialized in AL care since $>30$ years, described overall survival rates of 10.5 years after NTX and a 5-year transplant survival rate of $81 \%$ [46]. These data correspond to the outcome of NTX patients with other underlying kidney-specific diseases. NTX is therefore an adequate therapy for AL patients requiring dialysis. Especially since AL patients with chronic hemodialysis (CHD) report poorer life-quality and shorter life expectancy. In addition, during an observation period of $>7$ years, no increased incidence of lymphoproliferative diseases or other malignancies was found. An AL relapse after NTX can be treated safely and successfully.

Therefor AL patients should not be excluded from organ transplantations. If the clonal directed therapy (e.g. also ASCT) has achieved a good response or a complete remission contact should be made with a specialized center to clarify the possibility of NTX [47].

In MGRS clonal directed therapy and hematologic response should clearly precede NTX because recurrence of disease is unavoidable if the "toxic" paraprotein is not sufficient eradicated.

\section{Summary}

Systemic AL and MGRS are complex multisystem diseases. For the final and unambiguous diagnosis tissue specimen and intensive multimodal pathological processing is imperative. The implementation of riskadapted therapy regimens requires high levels experience and close interdisciplinary cooperation. Due to the rarity of the diseases and the complex modalities of organ damage, the patients with AL and MGRS should treated within a highly trained and specialized center. Currently in the U.S. the FDA approved daratumumab as the first drug for AL. No approved therapy is available in Europe. 


\section{Take home message}

- Awareness of AL and MGRS should be intensified and broadened.

- Early diagnoses and treatment are of major impact regarding improvement of organ function and overall survival in AL and MGRS.

- Treatment should be given in a risk-adapted manner.

- The management of treatment side effect and diseases related problems is complex and needs intensive interdisciplinary cooperation.

- Daratumumab is highly effective and well tolerated.

- Daratumumab is the first and only drug approved up to now (FDA approved since January 15, 2021).

Funding Open Access funding provided by Medical University of Vienna

Conflict of interest M. T. Krauth and H. Agis declare that they have no competing interests.

Open Access This article is licensed under a Creative Commons Attribution 4.0 International License, which permits use, sharing, adaptation, distribution and reproduction in any medium or format, as long as you give appropriate credit to the original author(s) and the source, provide a link to the Creative Commons licence, and indicate if changes were made. The images or other third party material in this article are included in the article's Creative Commons licence, unless indicated otherwise in a credit line to the material. If material is not included in the article's Creative Commons licence and your intended use is not permitted by statutory regulation or exceeds the permitted use, you will need to obtain permission directly from the copyright holder. To view a copy of this licence, visit http://creativecommons.org/licenses/by/4.0/.

\section{References}

1. Merlini G, Stone MJ. Dangerous small B-cell clones. Blood. 2006;108(8):2520-30. https://doi.org/10.1182/blood2006-03-001164.

2. Leung N, Bridoux F, Hutchison CA, Nasr SH, Cockwell P, Fermand JP, et al. Monoclonal gammopathy of renal significance: when MGUS is no longer undetermined or insignificant. Blood. 2012;120(22):4292-5.https://doi.org/ 10.1182/blood-2012-07-445304.

3. Leung N, Bridoux F, Batuman V, Chaidos A, Cockwell P, D'Agati VD, et al. The evaluation of monoclonal gammopathy of renal significance: a consensus report of the International Kidney and Monoclonal Gammopathy Research Group. Nat Rev Nephrol. 2019;15(1):45-59. https:// doi.org/10.1038/s41581-018-0077-4. Erratum in: Nat Rev Nephrol. 2019Feb;15(2):121.

4. Palladini G, Milani P, Merlini G. Management of AL amyloidosis in 2020. Blood. 2020;136(23):2620-7.https://doi.org/ 10.1182/blood.2020006913.

5. Amaador K, Peeters H, Minnema MC, Nguyen TQ, Dendooven A, Vos JMI, et al. Monoclonal gammopathy of renal significance (MGRS) histopathologic classification, diagnostic workup, and therapeutic options. Neth J Med. 2019;77(7):243-54. PMID: 31582582.

6. Popkova T, Hajek R, Jelinek T. Monoclonal antibodies in the treatment of $\mathrm{AL}$ amyloidosis: co-targetting the plasma cell clone and amyloid deposits. Br J Haematol. 2020;189(2):228-38. https://doi.org/10.1111/bjh.16436.

7. Milani P, Palladini G. Conventional therapy for amyloid light-chain amyloidosis. ActaHaematol. 2020;143(4):365-72. https:// doi.org/10.1159/000507072.

8. Oliva L, Orfanelli U, Resnati M, Raimondi A, Orsi A, Milan E, et al. The amyloidogenic light chain is a stressor that sensitizes plasma cells to proteasome inhibitor toxicity. Blood. 2017;129(15):2132-42. https://doi.org/10.1182/ blood-2016-08-730978.

9. Reece DE, Sanchorawala V, Hegenbart U, Merlini G, Palladini G, Fermand JP, et al. Weekly and twice-weekly bortezomib in patients with systemic AL amyloidosis: results of a phase 1 dose-escalation study. Blood. 2009;114(8):1489-97. https://doi.org/10.1182/blood2009-02-203398.

10. Reece DE, Hegenbart U, Sanchorawala V, Merlini G, Palladini G, Bladé J, et al. Efficacy and safety of once-weekly and twice-weekly bortezomib in patients with relapsed systemic AL amyloidosis: results of a phase 1/2 study. Blood. 2011;118(4):865-73. https://doi.org/10.1182/blood-201102-334227.

11. Phillips EH, Nash S, Adedayo T, Whelan CJ, Fontana M, MahmoodS, etal. Pitfalls in conducting prospective trials in stage III cardiac amyloidosis-experiencefrom the REVEAL study. Amyloid. 2017;24(4):242-4. https://doi.org/10. 1080/13506129.2017.1385453.

12. Dispenzieri A, Buadi F, Kumar SK, Reeder CB, Sher T, Lacy MQ, et al. Treatment of immunoglobulin light chain amyloidosis: mayo stratification of myeloma and riskadapted therapy (mSMART) consensus statement. Mayo Clin Proc. 2015;90(8):1054-81. https://doi.org/10.1016/j. mayocp.2015.06.009.

13. Manwani R, Cohen O, Sharpley F, Mahmood S, Sachchithanantham S, Foard D, et al. A prospective observational study of 915 patients with systemic AL amyloidosis treated with upfront bortezomib. Blood. 2019;134(25):2271-80. https://doi.org/10.1182/blood. 2019000834.

14. Basset M, Milani P, Nuvolone M, Benigna F, Rodigari L, Foli A, et al. Sequential response-driven bortezomib-based therapy followed by autologous stem cell transplant in AL amyloidosis. Blood Adv. 2020;4(17):4175-9. https://doi. org/10.1182/bloodadvances.2020002219.

15. Bochtler T, Hegenbart U, Kunz C, Granzow M, Benner A, Seckinger A, et al. Translocation $\mathrm{t}(11 ; 14)$ is associated with adverse outcome in patients with newly diagnosed AL amyloidosis when treated with bortezomib-based regimens. J Clin Oncol. 2015;33(12):1371-8. https://doi.org/10.1200/ JCO.2014.57.4947.

16. Dumas B, Yameen H, Sarosiek S, Sloan JM, Sanchorawala V. Presence of $\mathrm{t}(11 ; 14)$ in $\mathrm{AL}$ amyloidosis as a marker of response when treated with a bortezomib-based regimen. Amyloid. 2020;27(4):244-9. https://doi.org/10. 1080/13506129.2020.1778461. Epub 2020 Jun 19. PMID: 32551974

17. Palladini G, Milani P, Foli A, Obici L, Lavatelli F, Nuvolone M, et al. Oral melphalan and dexamethasone grants extended survival with minimal toxicity in AL amyloidosis: longterm results of a risk-adapted approach. Haematologica. 2014;99(4):743-50. https://doi.org/10.3324/haematol. 2013.095463.

18. Palladini G, Milani P, Foli A, Vidus Rosin M, Basset M, Lavatelli F, et al. Melphalan and dexamethasone with or without bortezomib in newly diagnosed AL amyloidosis: a matched case-control study on 174 patients. 
Leukemia. 2014;28(12):2311-6. https://doi.org/10.1038/ leu.2014.227.

19. Kastritis E, LeleuX, Arnulf B, Zamagni E, Cibeira MT, KwokF, etal. Bortezomib, melphalan, and dexamethasonefor lightchain amyloidosis. J Clin Oncol. 2020;38(28):3252-60. https://doi.org/10.1200/JCO.20.01285.

20. Manwani R, Mahmood S, Sachchithanantham S, Lachmann HJ, Gillmore JD, Yong K, et al. Carfilzomib is an effective upfront treatment in AL amyloidosis patients with peripheral and autonomic neuropathy. Br J Haematol. 2019;187(5):638-41. https://doi.org/10.1111/bjh.16122.

21. SanchorawalaV,PalladiniG, KukretiV,ZonderJA, CohenAD, Seldin DC, et al. A phase 1/2 study of the oral proteasome inhibitor ixazomib in relapsed or refractory AL amyloidosis. Blood. 2017;130(5):597-605. https://doi.org/10.1182/ blood-2017-03-771220

22. Dispenzieri A, Kastritis E, Wechalekar AD, Schönland SO, Kihyun K, Sanchorawala V, et al. Primary results from the phase 3 tourmaline-AL1 trial of Ixazomib-dexamethasone versus physician's choice of therapy in patients (pts) with relapsed/refractory primary systemic AL Amyloidosis (RRAL). Blood. 2019;134(Supplement_1):139. https://doi. org/10.1182/blood-2019-124409.

23. Kastritis E, Gavriatopoulou M, Roussou M, Bagratuni T, Migkou M, Fotiou D, et al. Efficacy of lenalidomide as salvage therapy for patients with AL amyloidosis. Amyloid. 2018;25(4):234-41. https://doi.org/10.1080/13506129. 2018.1540410.

24. Milani P, Sharpley F, Schönland SO, Basset M, Mahmood S, Nuvolone M, et al. Pomalidomide and dexamethasone grant rapid haematologic responses in patients with relapsed and refractory AL amyloidosis: a European retrospective series of 153 patients. Amyloid. 2020;27(4):231-6. https://doi.org/10.1080/13506129.2020.1767566.

25. Chakraborty R, Lentzsch S. Emerging drugs for the treatment of light chain amyloidosis. Expert Opin Emerg Drugs. 2020;25(3):299-317. https://doi.org/10.1080/14728214. 2020.1803829.

26. Sanchorawala V, Sarosiek S, Schulman A, Mistark M, Migre ME, Cruz R, et al. Safety, tolerability, and response rates of daratumumab in relapsed AL amyloidosis: results of a phase 2 study. Blood. 2020;135(18):1541-7. https:// doi. org/10.1182/blood.2019004436.

27. Palladini G, Kastritis E, Maurer MS, Zonder J, Minnema MC, Wechalekar AD, et al. Daratumumab plus CyBorD for patients with newly diagnosed AL amyloidosis: safety runin results of ANDROMEDA. Blood. 2020;136(1):71-80. https://doi.org/10.1182/blood.2019004460.

28. Parker TL, Rosenthal A, Sanchorawala V, Landau HJ, Campagnaro E, Kapoor P, et al. A phase II study of isatuximab (SAR650984) (NSC-795145) for patients with previously treated AL amyloidosis (SWOG S1702; NCT\#03499808). Blood. 2020;136(Supplement 1):20-1. https://doi.org/10. 1182/blood-2020-143180.

29. Premkumar V, Lentzsch S, Bhutani D. Single arm, prospective, open-label phase II trial to evaluate the efficacy of Isatuximab in patients with monoclonal gammopathy of renal significance. Blood. 2019;134(Supplement_1):3161. https://doi.org/10.1182/blood-2019-123496.

30. Wall JS, Kennel SJ, Williams A, Richey T, Stuckey A, Huang Y, et al. AL amyloid imaging and therapy with a monoclonal antibody to a cryptic epitope on amyloid fibrils. Plos One. 2012;7(12):e52686. https://doi.org/10.1371/journal.pone. 0052686.

31. Gertz MA, Landau H, Comenzo RL, Seldin D, Weiss B, Zonder J, et al. First-in-human phase I/II study of NEOD001 in patients with light chain amyloidosis and persistent organ dysfunction. J Clin Oncol. 2016;34(10):1097-103. https://doi.org/10.1200/JCO.2015.63.6530.

32. Gertz MA, Cohen AD, Comenzo RL, Du Mond C, Kastritis E, Landau HJ, et al. Results of the phase 3 VITAL study of NEOD001 (Birtamimab) plus standard of care in patients with light chain (AL) amyloidosis suggest survival benefit for mayo stage IV patients. Blood. 2019;134(Supplement_1):3166. https://doi.org/10.1182/blood-2019124482.

33. Edwards CV, Gould J, Langer AL, Mapara M, Radhakrishnan J, Maurer MS, et al. Interim analysis of the phase $1 \mathrm{a} / \mathrm{b}$ study of chimeric fibril-reactive monoclonal antibody 11-1F4 in patients with AL amyloidosis. Amyloid. 2017;24(sup1):58-9. https://doi.org/10.1080/13506129. 2017.1292900.

34. Bodin K, Ellmerich S, Kahan MC, Tennent GA, Loesch A, Gilbertson JA, et al. Antibodies to human serum amyloid P component eliminate visceral amyloid deposits. Nature. 2010;468(7320):93-7. https://doi.org/10.1038/ nature09494.

35. Richards DB, Cookson LM, Berges AC, Barton SV, Lane T, Ritter JM, et al. Therapeutic clearance of amyloid by antibodies to serum amyloid P component. N Engl J Med. 2015;373(12):1106-14. https://doi.org/10.1056/ NEJMoa1504942.

36. Richards DB, Cookson LM, Barton SV, Liefaard L, Lane T, Hutt DF, et al. Repeat doses of antibody to serum amyloid $\mathrm{P}$ component clear amyloid deposits in patients with systemic amyloidosis. Sci Transl Med. 2018;10(422):3128. https:// doi.org/10.1126/scitranslmed.aan3128.

37. GSK. 2019. https://www.gsk.com/en-gb/search. Accessed 11 Oct 2019

38. Kumar S, Kaufman JL, Gasparetto C, Mikhael J, Vij R, Pegourie B, et al. Efficacy of venetoclax as targeted therapy for relapsed/refractory $\mathrm{t}(11 ; 14)$ multiple myeloma. Blood. 2017;130(22):2401-9. https://doi.org/10.1182/ blood-2017-06-788786.

39. Muchtar E, Dispenzieri A, Kumar SK, Ketterling RP, Dingli D, LacyMQ, etal. Interphasefluorescencein situhybridization in untreated AL amyloidosis has an independent prognostic impact by abnormality type and treatment category. Leukemia. 2017;31(7):1562-9. https://doi.org/10.1038/ leu.2016.369.

40. PremkumarV, Bhutani D, Liedtke M. Venetoclax shows high efficacy in relapsed/refractory AL amyloidosis: a multicenter, international retrospective study. 17th International Symposium on Amyloidosis. 2020.

41. Ward JE, Ren R, Toraldo G, Soohoo P, Guan J, O'Hara C, et al. Doxycycline reduces fibril formation in a transgenic mouse model of AL amyloidosis. Blood. 2011;118(25):6610-7. https://doi.org/10.1182/blood-2011-04-351643.

42. Wechalekar AD, Whelan C. Encouraging impact of doxycycline on early mortality in cardiac light chain (AL) amyloidosis. Blood Cancer J. 2017;7(3):e546. https://doi.org/10. 1038/bcj.2017.26.

43. Hegenbart U. EGCG treatment in patients with cardiac AL amyloidosis: first results of a randomized and placebo-controlled clinical trial of GERAMY. International Symposium on Amyloidosis 2018. 2018.

44. Kristen AV, Kreusser MM, Blum P, Schönland SO, Frankenstein L, Dösch AO, et al. Improved outcomes after heart transplantation for cardiac amyloidosis in the modern era. JHeartLung Transplant. 2018;37(5):611-8. https://doi.org/ 10.1016/j.healun.2017.11.015.

45. Nuvolone M, Merlini G. Improved outcomes for kidney transplantation in AL amyloidosis: impact on practice. 
Kidney Int. 2019;95(2):258-60. https://doi.org/10.1016/j. kint.2018.11.003.

46. Angel-KormanA, Stern L, SarosiekS, Sloan JM, DorosG, Sanchorawala V, et al. Long-term outcome of kidney transplantation in AL amyloidosis. Kidney Int. 2019;95(2):405-11. https://doi.org/10.1016/j.kint.2018.09.021. Erratum in: Kidney Int. 2019Sep;96(3):796.

47. Sathick IJ, Rosenbaum CA, Gutgarts V, Landau H. Kidney transplantation in AL amyloidosis: is it time to maximize access? Br J Haematol. 2020;188(3):el-e4. https://doi.org/ 10.1111/bjh.16246.
Publisher's Note Springer Nature remains neutral with regard to jurisdictional claims in published maps and institutional affiliations.

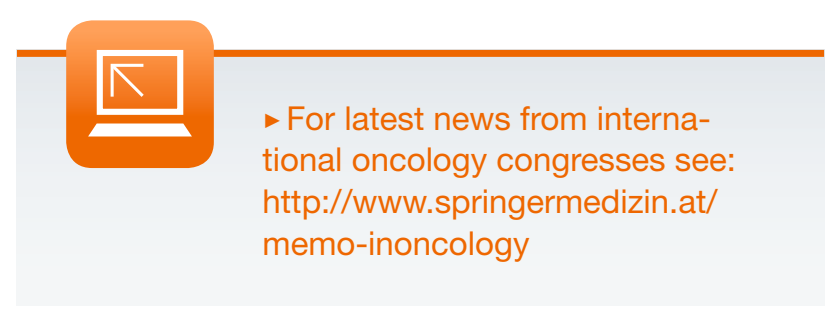

